\title{
El alimento más completo: debates y prácticas sobre el consumo de leche en México
}

\author{
The most nutritious food: \\ debates and practices about \\ milk consumption in Mexico
}

\author{
Sandra Aguilar Rodríguezi \\ ' Department of History/Moravian University. \\ Bethlehem - PA - USA \\ https://orcid.org/0000-0002-8771-9135 \\ aguilars@moravian.edu
}

Recebido em 20 nov. 2020.

Aprovado em 1 jun. 2021.

http://dx.doi.org/10.1590/S0104-59702021000400015

AGUILAR RODRÍGUEZ, Sandra. El alimento más completo: debates y prácticas sobre el consumo de leche en México. História, Ciências, Saúde Manguinhos, Rio de Janeiro, v.28, n.4, out.-dez. 2021, p.1201-1219.

\section{Resumen}

El presente texto explora el consumo de leche en la Ciudad de México entre 1930-1960 a partir de la implementación de políticas públicas y prácticas cotidianas. El texto da cuenta de las estrategias del estado mexicano para incrementar el consumo de leche mediante la importación de leche en polvo, su rehidratación y distribución a precios subsidiados. Médicos, nutriólogos y la industria lechera reprodujeron un discurso de superioridad de la leche, negando o cuestionando el valor nutricional de las dietas bajas en lácteos. Las historias de vida muestran la perspectiva de las mujeres y las dificultades en implementar los ideales de clase media en hogares campesinos y de clase trabajadora.

Palabras clave: nutrición; raza; políticas públicas; leche de vaca; historia.

Abstract

This text explores milk consumption in Mexico City from 1930-1960, starting with the implementation of public policies and day-to-day practices. The text examines the strategies employed by the Mexican state to increase milk consumption by importing powdered milk, rehydrating it and distributing it at subsidized prices. Doctors, nutritionists and the dairy industry reproduced a discourse of milk's superiority, denying or questioning the nutritional value of low-dairy diets. Life histories show women's perspective and the difficulties in implementing middle-class ideals in peasant and working-class homes.

Keywords: nutrition; race; public policies; cow's milk; history. 
Leche, cereales, legumbres, verduras, carne y frutas, formarán la base de la alimentación de la madre quien deberá tomar diariamente cuando menos un litro de leche.

Martínez Báez et al., 1933

En 1933 se publicó el Libro para la madre mexicana, escrito por un grupo de médicos, entre ellos Manuel Martínez Báez y Francisco de Paula Miranda. El objetivo del mismo era educar a las mujeres y reducir la tasa de mortalidad infantil además de mejorar la nutrición. ${ }^{1}$ Este libro, así como la mayoría de los médicos y especialistas en salud de la época, asumió que la dieta de los mexicanos debía cambiar para mejorar su salud e incrementar su productividad (Martínez Báez et al., 1933). El consumo de leche de vaca tuvo un papel central en el mejoramiento de la dieta ya que se veía como la fuente de proteína animal de más fácil acceso, pero también tenía un valor simbólico al ser un alimento civilizatorio que se identificaba con las naciones avanzadas del norte global, es decir Inglaterra, Francia y EEUU. El presente artículo analiza el consumo de leche entre 1930 y 1960 en zonas semirurales y urbanas de la Ciudad de México con base en el discurso médico, las políticas públicas y la publicidad producida por la industra lechera para compararlo con las prácticas cotidianas desde una perspectiva femenina. De esta manera, muestra como en estas décadas el Estado y la industria promovieron el consumo de leche y la identificaron con la salud y la clase media mestiza.

La clase media se identificó con profesionistas y trabajadores de cuello blanco mestizos que habitaban en centros urbanos y con familias donde la mujer no trabajaba fuera de casa. Por el contrario las clases bajas se asociaban con migrantes del campo y de origen indígena. Las mujeres tuvieron un papel central en los cambios alimenticios, ya que eran las encargadas de comprar y preparar los alimentos. Este trabajo parte de documentos del Archivo Histórico de la Secretaría de Salud, historias de vida realizadas en la Ciudad de México (CDMX), censos, revistas médicas, recetarios y prensa nacional. Las historias de vida consistieron en entrevistas a profundidad con duración entre una y dos horas con el fin de explorar el cambio en las prácticas alimenticias y percepciones de clase y género. ${ }^{2}$ Dichas historias nos ayudan a entender el consumo de leche en relación a lo que los médicos señalaban como prácticas ideales, además de proporcionar una perspectiva difícil de encontrar en documentos de archivo o revistas médicas.

Después de 1920 los gobiernos posrevolucionarios se avocaron a mejorar las condiciones de trabajo, educación y salud mediante la implementación de una serie de programas destinados a aquellos grupos a los que se veía como ignorantes por lo que los "expertos se consideraron con el derecho a educarlos mediante la modificación de sus prácticas cotidianas, alimenticias y de higiene" (Kapelusz-Poppi, 2001a, p.42). La nutrición fue un aspecto central para mejorar la salud y por ende la raza, pero la definición de una dieta ideal no se limitó a contar el número de calorías o vitaminas, sino que reprodujo una serie de valores culturales y morales de clase media (Roth, 2000; Cullather, 2007). Los discursos alimenticios de inicios del siglo XX no desaparecieron tras la Revolución como el presente texto muestra. Si bien se comprendió el valor de algunos alimentos, como el maíz, a mediados del siglo XX, la dieta de las clases populares continuó viéndose como deficiente. 
La leche de vaca se consideró un alimento esencial en el sano desarrollo tanto de la mujer embarazada como de la niñez. Los beneficios de la leche eran ensalzados por la industria, tanto en México como en EEUU. Organismos internacionales como el Fondo de las Naciones Unidas para la Infancia (Unicef) y la Organización de las Naciones Unidas para la Alimentación y la Agricultura (FAO) también promovieron el consumo de leche de vaca, particularmente entre los niños. Siguiendo esta idea, el Estado Mexicano buscó incrementar el consumo de leche a partir de 1940 con ayuda de la importación de leche en polvo proveniente principalmente de los EEUU para subsanar las baja producción y el impacto de la fiebre aftosa. El gobierno la reconstituía y distribuía como alimento subsidiado mediante tiendas del Estado, el programa de desayunos escolares y a través de comedores populares (Aguilar Rodríguez, 2007). Estas políticas muestran que tanto médicos expertos en nutrición como los creadores de políticas públicas adoptaron un discurso que reproducía la idea de la superioridad de la leche, negando o cuestionando, en el mejor de los casos, el valor nutricional de las dietas carentes de lácteos.

Partimos de la historiografía de género, raza y nutrición para reflexionar cómo la leche de vaca se identificó con ciertos grupos sociales y cualidades raciales. Cabe destacar los textos que han estudiado las políticas públicas de salud desde una perspectiva de género en el siglo XX (Stern, 1999; Bliss, 2001; Agostoni, 2002; Blum, 2004; Sanders, 2011). Dichos trabajos muestran la forma en que médicos, criminólogos y otros expertos, en su mayoría hombres, se avocaron a cambiar el comportamiento de las mujeres de clase baja a las que veían en riesgo de caer en la prostitución, además de considerar esencial el modificar las prácticas cotidianas de las madres de familia. Para ellos las mujeres requerían entrenamiento con base científica por lo que promovieron el estudio de la higiene, nutrición y puericultura. Sin embargo, poco se ha escrito sobre la agencia de las mujeres como objeto de intervención de programas de nutrición en particular en espacios privados.

El estudio de la alimentación desde una perspectiva histórica es aún más reciente que los estudios de género. En el caso de México inicia con el trabajo de Jeffrey Pilcher sobre identidad y comida, y ha continuado con el estudio histórico de la alimentación y su vinculación con la cultura, economía, política, ciencia y tecnología (Pilcher, 1996, 1998, 2006). Si bien contamos con estudios sobre la industria lechera y las políticas públicas en relación a la intervención del Estado, poco se ha escrito sobre el consumo cotidiano de leche de vaca en México (Ochoa, 1999; López Rosado, 1988; Saucedo Montemayor, 1984). Mas recientemente historiadores han explorado la intersección entre nutrición, ciencia y políticas públicas para mostrar la forma en la que el Estado se afanó en modificar la alimentación con fines eugenésicos y también para optimizar la producción de los trabajadores (Pío Martínez, 2013; Vargas Domínguez, 2015).

El interés en estudiar raza y racialización en México se ha incrementado en las últimas décadas. Si bien hasta hace poco la clase social se consideraba como el elemento definitorio, los estudios recientes muestran que el vincular clase social con raza nos ayuda a realizar análisis más complejos que desmontan la asunción de igualdad creada por la ideología del mestizaje (Stern, 2003; Sanders, 2017; Rosemblatt, 2018). La categoría de clase ha sido entendida desde una perspectiva económica, sin embargo la descripión de la clase media en particular no es un concepto fijo "sino una categoría de análisis cambiante en 
función de múltiples variables, como pueden ser el gusto, el poder adquisitivo, las formas de sociabilidad, los espacios de reunión, las profesiones, entre otros" (Sánchez Parra, Matamoros, 2020, p.6). ${ }^{3}$ Por su parte, el concepto de raza va más allá de lo biológico y se convierte en una cuestión de prácticas culturales y aspiracionales vinculadas a la clase. Este trabajo parte de este horizonte al presentar una perspectiva distinta sobre los discursos, políticas y programas de bienestar social que buscaron incrementar el consumo de leche. El estudio de dicho discurso nos muestra las dinámicas de poder en las que si bien los médicos detentaron la legitimidad, fueron las mujeres, madres y amas de casa, quienes aceptaron, rechazaron o adaptaron el consumo de leche en su vida cotidiana.

\section{Discursos de raza y nutrición}

Tras la contienda revolucionaria y debido a la posterior industrialización del país se incrementó la migración del campo a la ciudad, lo que conllevó al crecimiento de las urbes, particularmente Ciudad de México. La situación en el campo distaba considerablemente del ideal citadino debido a la carencia de servicios básicos como electricidad, agua potable y centros médicos (Vaughan, Fowler-Salamini, 1994). Bajo estas condiciones, en la década de 1930, el Estado mexicano comenzó a sistematizar sus políticas de nutrición a partir de la idea de que la alimentación era un elemento fundamental para mejorar la raza e incrementar la productividad. Las discusiones sobre el mejoramiento de la raza estuvieron influenciadas por el desarrollo de la eugenesia y el racismo científico en Europa y EEUU. Francis Galton estableció la disciplina llamada eugenesia con el fin de estudiar el mejoramiento de la raza humana. Galton asumió que aquellos individuos o grupos que disponían de un material genético adecuado eran sanos, inteligentes y exitosos independientemente del contexto socioeconómico en el que vivían. La postura de Galton predominó en países como EEUU, Gran Bretaña y Alemania ya que sus habitantes se consideraban a sí mismos como herederos de una raza superior y por ende con el derecho y la legitimidad de dominar el mundo. Ante esta postura se desarrolló un contradiscurso en los países latinos inspirado en el trabajo de Jean-Baptiste Lamarck, quien propuso la teoría de la herencia de los caracteres adquiridos (Suárez y López Guazo, 2005; Stern, 2016).

El Lamarckismo implicó la oportunidad de mejorar la raza a través de modificaciones a las condiciones materiales y sociales. Por lo que la educación, la higiene, y los cuidados a la salud se convirtieron en el centro de esta vertiente (Stepan, 1991; Bliss, 2001). El Lamarckismo influenció a intelectuales y médicos mexicanos quienes adoptaron dicha postura particularmente después de la Revolución. Los pobres, palabra que se identificó con los grupos indígenas o campesinos, no estaban en esa situación por ser flojos o por pertenecer a una raza inferior; sino por las condiciones materiales en las que vivían, la falta de higiene, la malnutrición y la ignorancia (Stepan, 1991). A partir de 1930 dicha interpretación fue también influenciada por el marxismo dando mayor importancia al aspecto económico (Rosemblatt, 2018). La raza se entendió como un conjunto de características físicas y morales que podían modificarse.

Los gobiernos posrevolucionarios adoptaron el discurso del mestizaje y lo combinaron con el indigenismo. Así se exaltó la mezcla entre la herencia española e indígena y se 
intentó proteger algunos aspectos de la cultura indígena (Rosemblatt, 2018). Si bien no había cualidades biológicas que hiciera a un grupo mejor que otro, continuó identificándose a campesinos e indígenas con el atraso. El mejoramiento de la raza, al que muchas veces se refería como "civilizar" al pueblo mexicano, partía de mejorar la salud mediante la alimentación (Biltekoff, 2002). Esto implicó la adopción de una dieta identificada con países avanzados como los EEUU. El cambio en la alimentación iba de la mano de un cambio en los valores y costumbres ya que las campañas tanto de salubridad como educativas incentivaban la ética del trabajo, la disciplina, la limpieza y la abstinencia del alcohol. Se asumía que si las clases bajas modificaban su dieta y su comportamiento serían más productivas ya que no se enfermarían tanto y así no faltarían al trabajo. Además, siguiendo el Lamarckismo, transmitirían esa salud y esos valores a su descendencia lo cual conllevaría a mejorar la raza y el futuro del país. Los expertos y creadores de políticas públicas creían que la selección natural favorecería a aquellos individuos mejor adaptados, más eficientes y productivos, lo cual impulsaría un orden capitalista (Rosemblatt, 2018). Por ello la alimentación y la salud pública se veían como los elementos fundamentales para transformar a México en un país moderno e introducir a una economía de mercado a las comunidades indígenas y rurales.

El México de mediados del siglo XX se identificó con los espacios urbanos y una cultura de clase media que reprodujo sus valores e ideales a través de los medios masivos de comunicación y del desarrollo de una cultura del consumo (Niblo, 1999). La clase media inició como un grupo que buscó diferenciarce de los más pobres y parecerse a los más ricos y poco a poco fue creciendo al consolidarse la burocracia e identificarse con la modernización y el crecimiento económico (Barbosa Cruz, 2020). En la década de 1940 se implementó el programa de sustitución de importaciones para incrementar el crecimiento económico mediante la inversión nacional y extranjera. El Estado se comprometió a mantener salarios bajos, entre otras cosas, lo cual mitigó mediante programas de educación, bienestar social y alimentos subsidiados para mejorar las condiciones de vida de la población. De este modo se garantizaron ganancias altas para los inversionistas y la creación de empleo particularmente en los centros urbanos. Médicos, enfermeras, trabajadoras sociales, maestras, recetarios y revistas femeninas se avocaron a informar a las mujeres como mejorar sus condiciones de vida mediante la adopción de dietas balanceadas de bajo costo, además de promover valores de clase media (Aguilar Rodríguez, 2008).

El conocimiento sobre nutrición y economía doméstica buscó profesionalizar a las amas de casa sin cuestionar el patriarcado ya que las mujeres continuaron siendo las principales responsables de su hogar, de alimentar a su familia y cuidar a los niños. Si bien esta profesionalización incrementó la presencia de mujeres en espacios públicos al participar como maestras, enfermeras o trabajadoras sociales, las jerarquías sociales y los roles de género se mantuvieron al ser médicos hombres en su mayoría los que generaban el conocimiento y creaban los programas de nutrición, mientras que las encargadas de su implementación eran en su mayoría mujeres. Así el Estado posrevolucionario trató de ocupar el lugar del padre en el hogar para convertirse en el paterfamilias controlando tanto la esfera pública como privada (Vaughan, 1977). 


\section{Institucionalización de los programas de nutrición}

En 1936 se fundaron la Oficina General de Higiene de la Alimentación y la Comisión Nacional de Alimentación, bajo la tutela del Departamento de Salubridad Pública, para crear programas de nutrición. El doctor José Quintín Olascoaga fungió como director de la Comisión y de la Sección de Investigación de la Alimentación Popular perteneciente a la Oficina General de Higiene de la Alimentación, y fue quien propuso la sistematización de los estudios sobre nutrición (Quintín Olascoaga, 1948; Alvarez Amézquita, 1960; Gutierrez de Olmo, 1993). Con dicho fin, en 1943, se creó el Instituto Nacional de Nutriología (INN) al mando del doctor Francisco de Paula Miranda, quien estableció intercambios con instituciones nacionales e internacionales (Arroyo, 2006). En pocos años el INN se convirtió en "un centro de investigación de punta en el terreno de la alimentación" (Vargas Domínguez, 2019, p.513). Para Miranda, los problemas de nutrición eran causados por la pobreza y a esta perspectiva le llamó nutrición social. Para mejorar la dieta era necesario incrementar el salario o bien reducir el costo de los alimentos con "mecanismos como el aumento de la producción, el control de precios y la mejoría de los canales de distribución" (Vargas Domínguez, 2019, p.519). Las políticas de nutrición no solo se centraron en mantener los bajos costos y subsidiar alimentos, sino también en la promoción de ciertos alimentos como lo fue la leche. El Estado apostó a la producción, al subsidio de precios y al incremento del acceso a productos de alimentación básicos en vez de aumentar los salarios. Debido a restricciones económicas, el INN inició sus programas en la CDMX para posteriormente expandirse al resto del país.

La CDMX fue un buen sitio para iniciar ya que poseía la infraestructura necesaria, pero también una gran desigualdad social. Tras la Revolución y las políticas de sustitución de importaciones, la capital atrajo migrantes de toda la nación por lo que pasó de 1.757.530 habitantes en 1940 a 3.050.442 en 1950. Si bien la capital era principalmente urbana, en 1950 las zonas aledañas eran espacios rurales y sus habitantes representaban el 5,4\% del total de la población. A pesar de la migración del campo a la ciudad, en 1950 aún la mayoría de los mexicanos vivían en pueblos y caseríos de menos de 2.500 habitantes, representando el 57,4\% del total de la población nacional (DGE, 1950; INEGI, 1998). La mayoría de los migrantes eran campesinos que vivían hacinados en vecindades en el centro de la ciudad o bien en las cercanías de la zona industrial situada al norte. A su llegada, la mayoría se empleaba en fábricas, como vendedores ambulantes o empleadas domésticas.

El gobierno trató de facilitar la transformación de campesinos a obreros mediante programas educativos y de bienestar social. La comunidad médica sugirió que el ausentismo laboral era causado en parte por enfermedades relacionadas a la mala nutrición y la poca higiene que se identificaba con los hogares de bajos recursos. Aún los trabajadores que pocas veces faltaban, según los médicos, tenían una baja productividad causada por una dieta insuficiente. Un porcentaje de las enfermedades más comunes entre las clases bajas (parasitosis, diarrea, anemia y pelagra) eran causadas por una mala nutrición y falta de higiene y representaban el 18,5\% del total de muertes (DGE, 1950). Por ello, el Estado se dio a la tarea de enseñar a los migrantes del campo, muchos de origen indígena, cómo vivir y organizar sus hogares teniendo como modelo a la clase media identificada con la cultura 
mestiza. Los cambios en la dieta no solo implicaron la adopción de nuevos ingredientes o técnicas de cocina, sino también las costumbres y percepciones de clase media como lo fueron comer en una mesa en vez de en un petate al ras del suelo y utilizar cubiertos en vez de solo tortillas para llevarse los alimentos a la boca. Incrementar el consumo de proteína animal fue considerado uno de los principales objetivos de los programas de bienestar social. Dentro de la proteína animal se vio a la leche de vaca como la fuente ideal debido a su bajo costo en comparación con la carne.

En el siglo XIX la leche de vaca se convirtió en un símbolo de modernidad y salud a la vez que su consumo incrementó en el norte global, particularmente en EEUU. De acuerdo con Melanie DuPuis (2002), en EEUU, la leche de vaca ha sido considerada como un "alimento perfecto" al ser presentada como superior a cualquier otro por su valor nutricional y por su importancia en la dieta de la civilización occidental. "La perfecta blancura de este alimento y el cuerpo blanco genéticamente capaz de digerirla en grandes cantidades se han identificado el uno con el otro. Al declarar a la leche como un alimento perfecto, los blancos del norte de Europa declaran su propia perfección," (DuPuis, 2002, p.11) dejando fuera a la mayoría del mundo y particularmente a los grupos que son intolerantes a la lactosa. La mayoría de la población de origen asiático, africano e indígena carece del "gen que permite producir lactasa, enzima que ayuda a digerir la lactosa tras la infancia ... de acuerdo con los estimados, dos tercios de la humanidad no pueden digerir la lactosa después de los seis años de edad" (p.27-28).

La población que puede digerir la lactosa es la que históricamente ha dependido de la leche para su supervivencia como es el caso de los nómadas del Sahara, quienes solo beben leche y sangre durante nueve meses, además de los habitantes de las zonas gélidas del norte de Europa, quienes tenían menos acceso a gran variedad de alimentos particularmente en los meses fríos (DuPuis, 2002). El consumo de leche líquida representó un papel menor en la dieta de la humanidad hasta tiempos modernos. En el siglo XX, la leche de vaca se convirtió en un alimento esencial para el desarrollo físico y mental de la población y por ende para su mejoramiento. La leche se asoció con la modernización, no solo por su valor nutricional sino también por el conocimiento y la tecnología que se desarrolló con el fin de industrializarla. El incremento en el consumo de leche en EEUU resultó de la construcción de vías férreas, el descubrimiento de la refrigeración y la pasteurización además de otras tecnologías. México trató de seguir el ejemplo de EEUU, pero la falta de recursos e infraestructura como vagones de tren refrigerados, hizo más lento y menos uniforme el crecimiento del mercado de la leche. La construcción de caminos incrementó tras la Revolución, en particular en la década de 1940, lo que poco a poco facilitó el traslado de la leche (Bess, 2017).

\section{La leche como alimento de lujo}

En la década de 1920 la leche de vaca se comenzó a presentar en México como un alimento altamente nutritivo y esencial en el desarrollo de la infancia. La leche embotellada y pasteurizada apareció en el mercado mexicano en 1910. "Sin embargo, los médicos afirmaron que el Departamento de Salubridad había descubierto la existencia de leches pasteurizadas con 
abundantes gérmenes" (López Fermán, 2007, p.208), por lo que se continúo recomendando comprar leche recién ordeñada. Las leches evaporada, condensada y en polvo se introdujeron en México antes de 1920, pero poco se conoce de su consumo ya que los médicos no hicieron mención sobre esos productos. En México había pocos establos, con vacas mal alimentada y en condiciones poco higiénicas por lo que la leche de vaca era escasa, cara y de baja calidad. En 1929 el doctor Ignacio Chávez creó la Gota de Leche para proveer de leche pura a niños de bajos recursos como parte del Servicio de Higiene Infantil. Dicho programa se encontraba inspirado en La Goutte de Lait, creada en Francia por Pierre Budin y otras semejantes en EEUU e Inglaterra. Sin embargo, la Gota de Leche desaparece tras 1931 (Viesca Treviño, 2008).

En 1936, tres años después de que el Libro para la madre mexicana recomendara el consumo de un litro de leche al día a las mujeres embarazadas, un reporte publicado por la Secretaría de Economía señaló que tres cuartas partes de los capitalinos no tomaban leche (del Bajío, 1990). La gente no compraba leche porque no estaba disponible o por su alto precio. Casi diez años después la situación seguía casi igual. En 1945 un litro de leche costaba 47 centavos en los establos, 54 en las tiendas y 56 si era entregada a domicilio (de la Peña, 8 ene. 1945). Si consideramos que el salario mínimo en 1945 era de \$1,65 peso en zonas rurales y $\$ 1,90$ peso en las ciudades, una familia obrera hubiera tenido que gastar casi un tercio de su salario para comprar un litro de leche (Presidencia de la República, 1963; Ochoa, 1999). Tanto los doctores Jesús Díaz Barriga como José Quintín Olascoaga, quienes trabajaban en el INN, dieron cuenta del bajo consumo de leche en México.

Díaz Barriga trabajó de manera cercana con Lázaro Cárdenas, quien fue gobernador del estado de Michoacán (1928-1932) y luego presidente de la República (1934-1940). Creó e implementó un innovador sistema de medicina rural y social (Kapelusz-Poppi, 2001a). Durante la presidencia de Cárdenas, Díaz trabajó como su asesor y fue delegado del Departamento de Salubridad en Morelia (Kapelusz-Poppi, 2001b). Después de 1943, tuvo un papel activo en el INN y, en 1950, era delegado de la SSA ante la FAO (Díaz Barriga, 14 feb. 1950). Quintín Olascoaga es considerado uno de los pioneros en estudios de dietética en México. En 1934 participó en las misiones culturales urbanas, llevadas a cabo por la Secretaría de Educación Pública. En 1935, se unió al Hospital General, a cargo de un grupo de investigación de alimentación popular, y fue cofundador del INN y director de la Escuela de Dietética (Quintín Olascoaga, 1948).

Díaz señaló que, en 1945, el 82\% de la población tenía pocos recursos económicos ya que ganaba menos de $\$ 600$ pesos al mes y gastaba al menos la mitad de sus ingresos en comida (Díaz Barriga, 18 nov. 1949). Este grupo, conformado por campesinos, obreros, artesanos, pequeños comerciantes y oficinistas de bajo rango, bebía muy poca leche (Díaz Barriga, Quintín Olascoaga, 1949). La mayoría de los campesinos no bebían leche, a menos de que vivieran en zonas ganaderas y aún así el consumo de leche estaba reservado para aquellos con mayores recursos económicos.

De acuerdo con los censos de 1950 y 1960 el consumo de leche de vaca era más común en las zonas urbanas que en las zonas rurales. Las leches consensada y evaporada se consumían particularment en los hogares de clase media (Banco de México, 1963). ${ }^{4}$ En la CDMX, tanto las clases medias como las altas compraban leche evaporada, sin embargo, su consumo en comparación con el de leche fresca seguía siendo bajo. En la capital representaba 3,5\% del 
total de gasto en productos lecheros (Banco de México, 1963). ${ }^{5}$ Si bien las zonas urbanas tenían mejor acceso a la leche debido a la existencia de establos además de contar con una mejor infraestructura para distribuirla y mantenerla refrigerada, su consumo dependía en gran medida del ingreso familiar. Las historias de vida de las mujeres entrevistadas para esta investigación confirman que las clases bajas, aquellas con un limitado ingreso, tenían un muy bajo consumo de leche y en muchos casos era nulo.

Carolina Basave nació en 1930 y pasó su infancia en una zona rural de Tlalpan, ubicada al sur de la CDMX. A inicios de los años 1930 la mamá de Carolina dejó a su marido y se fue a vivir con una de sus hermanas a las afueras de la ciudad. Su situación económica era muy difícil, su mamá se dedicaba a lavar ajeno y su dieta se limitaba a lo que podían sembrar y recolectar. Carolina cuenta que casi no tomaba leche y cuando así era, solo le agregaba un chorrito a otras bebidas como el café (Basave Morales, 31 ago. 2005). Crispina Vargas nació en 1935 y también vivía a las afueras del sur de la ciudad, en San Nicolás Totolapa. Su padre era albañil, pero abandonó a su madre poco después de que ella nació. La mamá de Crispina era trabajadora doméstica de tiempo completo por lo que Crispina y su hermano crecieron con sus abuelos maternos. En 1940, Crispina y su hermano se mudaron al centro de la ciudad con su mamá y su padrastro. Crispina nunca tomó leche durante el tiempo que vivió en San Nicolás, solo café negro. Bebió leche por primera vez cuando se fue a vivir con su mamá, quien compraba leche en un establo ubicado en la colonia Juárez muy cerca del centro. La hervían y la guardaban debajo de la mesa de la cocina ya que no tenían refrigerador (Vargas Romero, 19 ago. 2005). La leche, como muchos otros alimentos, tenía que ser comprada de manera cotidiana para mantenerla fresca en el caso de los hogares de clase baja que no contaban con refrigerador, los cuales constituían la mayoría en las décadas de 1940 y 1950.

La leche de vaca era parte de la dieta de las familias de clase media. Evelia Estrada nació en 1925 y creció en una vecindad del centro histórico, tuvo dos hermanos pero murieron en la infancia así que creció como hija única. Su padre murió cuando era niña, sin embargo, su tío, un sastre profesional de gran reputación con el que trabajaba su madre, les apoyó económicamente. Ella recuerda que cuando iba a la escuela preparatoria, a inicios de 1940, siempre tomaba leche en su desayuno que consistía en: "avena en leche una taza como de medio litro, dos huevos tibios, fruta o jugo y dos piezas de pan dulce con mantequilla y algo" (Estrada de Trejo, 1 sept. 2005). Tras concluir la preparatoria Evelia estudió en la Facultad de Medicina, pero poco después de graduarse se casó y dejó de trabajar para dedicarse al hogar.

Entre 1930 y 1950 el consumo de leche continuó implicando un riesgo pues frecuentemente la leche era adulterada. En diciembre de 1941, Faustino Vidal, director del Comedor Familiar $\mathrm{n}^{\mathrm{o}} 1$, localizado en el centro de la CDMX, reportó que la leche frecuentemente se cuajaba argumentando su mala calidad (Vidal, 4 dic. 1941; Aguilar Rodríguez, 2007). El 3 de diciembre de 1945, el doctor Samuel de la Peña, jefe del Control Sanitario de la Leche y sus Derivados bajo la Secretaría de Salubridad y Asistencia (SSA), afirmó que los periódicos estaban reportando casos de intoxicación y que sospechaban de las deplorables condiciones sanitarias de las lecherías. De la Peña (3 dic. 1945) señaló: “En pasada inspección sanitaria se consignó cierta cantidad de leche y otras substancias. La leche resultó adulterada por adición de sacarosa. Entre las substancias remitidas se encuentra cierta cantidad de moscabado, es decir, mezcla de sal común, glucosa, con huellas de sosa [sic] cáustica". 
La SSA llamó a todas sus dependencias a mantener vigilancia en las lecherías. Dicha medida, junto con otras implementadas por el INN, buscaban mejorar la calidad de la leche e incrementar su consumo. Tanto médicos como la alta burocracia encargada de crear políticas públicas dieron una gran importancia a la generalización del consumo de leche, ya que consideraban que transformaría a México para bien. Dicho discurso era parte de la tendencia a identificar la dieta del norte global con un alto valor nutricional, con salud, modernidad y progreso. Por ello, a pesar de que en México había otras fuentes proteínicas como los frijoles e insectos en ciertas regiones, la leche se consideró como la mejor alternativa.

\section{Los debates sobre la leche}

En la década de 1940, la SSA y el INN se avocaron a mejorar la alimentación del mexicano. Cada año morían alrededor de 125 de cada mil niños en su primer año de vida, 34 de estas muertes se debían a enfermedades de origen gastrointestinal mientras la anemia materno infantil era la causa de otras enfermedades mortales (INEGI-DGE, 1996). De acuerdo con los expertos:

Los alimentos de origen animal como son la leche, el huevo, la carne, el queso, la sangre de los animales, el pescado etc., son completamente indispensables para la alimentación humana, pues de allí va a depender la potencia de la raza ... Todos los pueblos que han sido grandes introducen en su dieta gran cantidad de estas proteínas. No hay carnívoro en la naturaleza que carezca de sagacidad, de inteligencia, de fuerza. Jamás podremos ser un pueblo superior, mientras 15 millones de mexicanos se alimenten a base de proteínas que son inadecuadas aún para bestias. El asunto de partida para levantar la Nación Mexicana será modificar profundamente la alimentación de todos nuestros niños, introduciéndoles a toda costa cualquier alimento de origen animal (El problema..., 1948).

Así médicos compararon al pueblo mexicano con aquellos que "han sido grandes", es decir con la cultura y economía del norte global, y en particular con los EEUU, Francia e Inglaterra. Dicho cambio debía ser introducido primero en los niños, lo cual se buscaría lograr con la introducción de leche en polvo. La producción de leche fresca era muy baja y tendía a ser adulterada además de que su costo era prohibitivo para la mayoría. Por otro lado, entre 1946 y 1955, la fiebre aftosa llevó a miles de vacas a ser sacrificadas. Por ello, para poder proveer al pueblo de leche a bajo costo, el gobierno incrementó las importaciones de leche en polvo en particular proveniente de EEUU (Saucedo Montemayor, 1984; Ochoa, 2000). Tanto en México como en EEUU dicha leche era una opción para las madres que no podían amamantar a sus hijos y que no tenían acceso a leche fresca (DuPuis, 2002; Schmid, 2003). Las ventajas de la leche en polvo eran su bajo costo, pureza, conveniencia y practicidad al no requerir refrigeración. Sin embargo, el no tener acceso a agua potable para prepararla resultaba un riesgo mayor para aquellos que la bebían. Desde la década de 1930, Nestlé importaba leche en polvo producida en los EEUU por Gail Borden. En los años 1940, los anuncios de Nestlé, Dry Co, The Eagle, Carnation, Sheffield, Borden, Klim y Valle Verde se encontraban con frecuencia en las revistas femeninas (Nestlé..., 2001). 


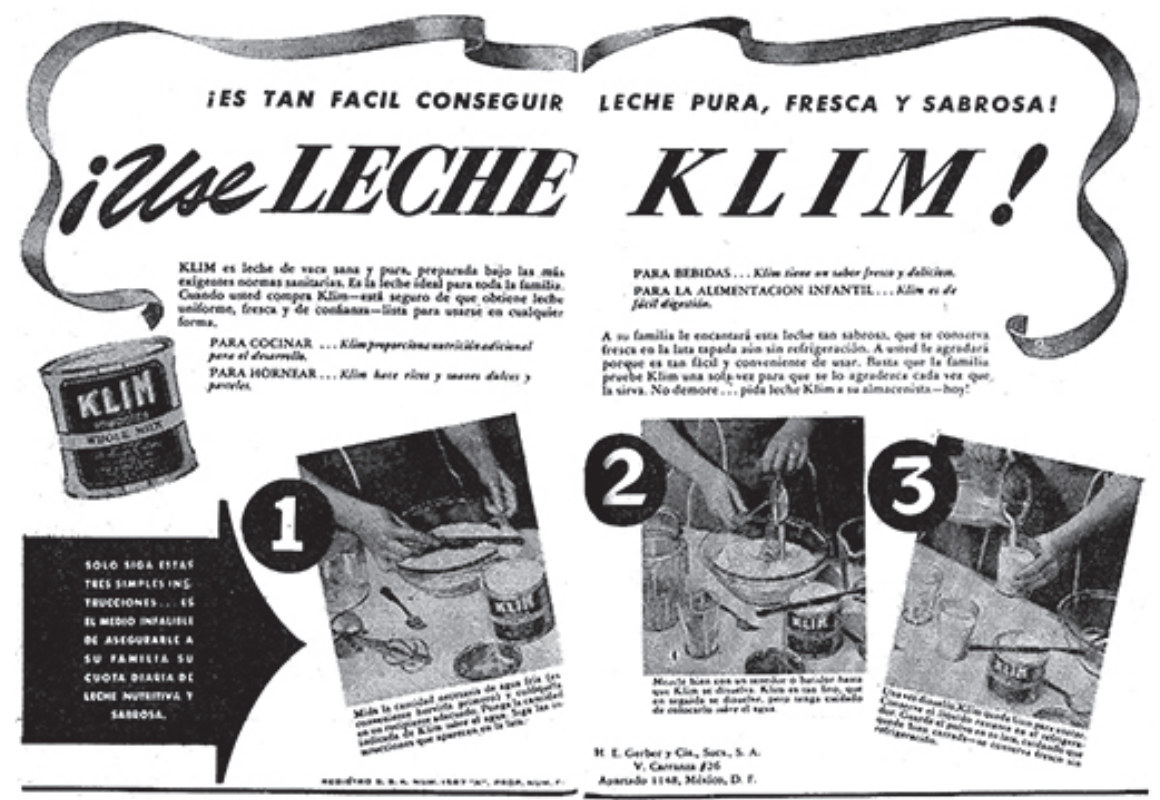

Figura 1: Leche Klim (Enciclopedia del Hogar, 1946)

Klim se presentaba como leche pura y fresca, además afirmaba que al comprar "leche Klim está seguro de que obtiene leche uniforme, fresca y de confianza". Por su parte, leche Valle Verde se presentaba como leche de vacas "que veterinarios afamados conservan en perfecto estado de salud ... pasa por todos los procesos de la producción y elaboración sin que la toquen manos humanas" (Yturbide de Limantour, 1947, p.27). De este modo se enfatizó no solo la pureza de raza de las vacas, sino también el conocimiento técnico de aquellos que las cuidaban. A cargo de las vacas no estaba el campesino o ranchero que carecía de estudios profesionales, sino los médicos veterinarios que vigilaban la salud de los animales. Además, la producción lechera era completamente mecanizada, lo que hacía que no entrara en contacto con manos humanas en ninguna etapa del proceso. Dicha publicidad afirmaba que leche Valle Verde "sabe a leche natural", no obstante la mayoría de las mujeres no pensaban que la leche en polvo fuera un sustituto de la leche fresca. También en el gremio médico había dudas sobre la superioridad de la leche reconstituida. El consenso estaba en su uso como una solución temporal.

El 15 de abril de 1944, antes del brote de fiebre aftosa, el doctor Demetrio Mayoral Pardo, director de la Dirección General de Higiene de Alimentos y Control de Medicamentos, escribió un memorándum enfatizando "toda medida que tienda a facilitar la importación de leches estabilizadas que llenen los requisitos que exigen nuestros reglamentos, es de aceptarse como buena, por lo menos mientras pasa el período de emergencia" (Mayoral Pardo, 15 abr. 1944). La leche evaporada y en polvo no es "un equivalente absoluto de la leche fresca" debido a que perdía vitaminas durante el proceso de evaporación. Mayoral agregó que "varios autores señalan el peligro potencial del uso prolongado de alimentos enlatados", además que a mucha gente no le gustaba su sabor. 
Un día antes, el 14 de abril, el Departamento de Producción y Provisión de Leche bajo el control de la SSA afirmó que se importaría leche de Argentina y EEUU. El 12 de diciembre, el periódico Excélsior publicó que el Departamento del Distrito Federal había firmado un acuerdo con EEUU para importar cien mil latas de leche evaporada con el fin de normalizar la distribución de leche en la capital que había disminuido por la fiebre aftosa (Excélsior, 12 dic. 1944). Mientras tanto, el periódico El Nacional (5 ago. 1945) reportó que México importaría cinco millones de latas de leche evaporada de vacas jersey: "Sabemos que este producto está siendo usado por las Naciones Unidas y sus ejércitos con magníficos resultados (se venderá) a precio bajo, o sea que la leche evaporada tendrá un costo aproximado por litro de veinticinco o treinta centavos, más bajo que la leche natural" (Se importarán..., 12 dic. 1944). Ocho meses después, El Nacional (5 ago. 1945) afirmó que "se ha comprobado que esta leche tiene mayores propiedades y mejor calidad que la leche líquida".

Además de la importación de leche evaporada, se establecieron en el país plantas hidratadoras de leche en polvo. Excélsior incluyó una nota el 15 de marzo de 1945 sobre la inauguración de una planta lechera en Lagos de Moreno, Jalisco, donde se elaboraban leches condensada y evaporada. El 9 de octubre de 1945, El Universal publicó un artículo en el que se aseguraba que "el alimento no solo no perderá en sus cualidades vitamínicas, sino que el sabor será idéntico, una vez salido de las plantas hidratadoras, al que tiene cuando es fresco y recién salido de la ordeña" (Igual..., 9 oct. 1945). La leche reconstituida se veía como de mayor calidad que la leche promedio disponible en zonas urbanas, la cual estaba diluida o hasta contaminada. Sin embargo, esta leche solo era para consumo de las clases bajas, ya que las clases medias y altas seguían prefiriendo la leche fresca. De ahí que la leche evaporada o en polvo se identificara con los estratos bajos de la población.

La prensa nacional promovió el consumo de leche afirmando el consenso internacional que presentaba a la leche de vaca como la mejor fuente de proteína. Tras el brote de fiebre aftosa, la importación de leche evaporada creció de $4.944 .901 \mathrm{~kg}$ en 1946 a $13.280 .891 \mathrm{~kg}$ un año después. Para 1958, la cantidad de leche en polvo que se importaba era alrededor de 25 toneladas (Ochoa, 2000). En 1949, la FAO publicó un folleto llamado Mejor aprovechamiento de la leche, en el que afirmaba: "La concentración de la leche constituye un método muy eficiente de preservación. Los productos pueden fácilmente transformarse en líquido mediante la adición de agua, casi sin menoscabo de su valor nutritivo. Por lo tanto, son excelentes substitutos de la leche fresca en aquellos lugares donde ésta no puede obtenerse" (FAO, ago. 1949). Tanto la FAO como la Unicef apoyaron el consumo de leche reconstituida, en ocasiones proveyéndola, por lo que estaban interesadas en desmentir cualquier duda sobre el valor nutricional de dicha leche (De la Torre et al., abr. 1950 ). No obstante, algunos doctores no estaban tan convencidos. En 1950, el doctor Díaz Barriga afirmó en la sección de nutrición de El Universal que "lo mejor es comprar leche fresca de vaca y hervirla bien, pero en el caso de que no sea posible comprarla pueden tomar leche en polvo descremada, que está a mitad de precio que la fresca" (Las condiciones..., 1950). Díaz invitaba a las mujeres a pedirle a los maestros que intercedieran con los comerciantes locales para que pusieran al alcance de la comunidad leche en polvo o evaporada.

El Estado favoreció la creación de plantas rehidratadoras de leche, destacando el caso de Lechería Nacional creada en 1946 por un grupo de empresarios mexicanos para producir Leche 
Sello Azul rehidratando, leche en polvo importada de los EEUU y mezclada con aceite de coco o algodón de $36 \mathrm{~g}$ por litro (Autret, 11 nov. 1951). Para 1951, la mitad de la leche que se vendía en la ciudad de México era producida por Lechería Nacional. El litro se vendía en 66 centavos, mientras que el litro de leche fresca costaba entre $\$ 1,10$ y $\$ 1,30$ peso (Autret, 11 nov. 1951). Sin embargo, la publicidad de leche Sello Azul, como lo muestra la Figura 2, afirmaba que la crema no ha sido separada "sino que está integrada en cada gota". La leche se describía como completa, es decir, leche con la grasa natural sin hacer mención al aceite de coco o algodón.

La publicidad presenta una familia de clase media donde el padre viste traje y corbata, lo cual apunta a que es un profesionista y el encargado de traer el pan a la casa, mientras que la madre lleva su delantal y sirve el desayuno tomando el rol de ama de casa. La pareja tiene tan solo dos hijos, un número muy reducido para la época. Los niños muy bien vestidos están sentados a la mesa esperan su vaso de leche. El fenotipo de la familia en nada se asemeja a la mayoría de la población mexicana, parece más bien sacada de una revista estadounidense, además de identificar el consumo de leche a un estrato social medio alto y no con personas de origen campesino o indígena.

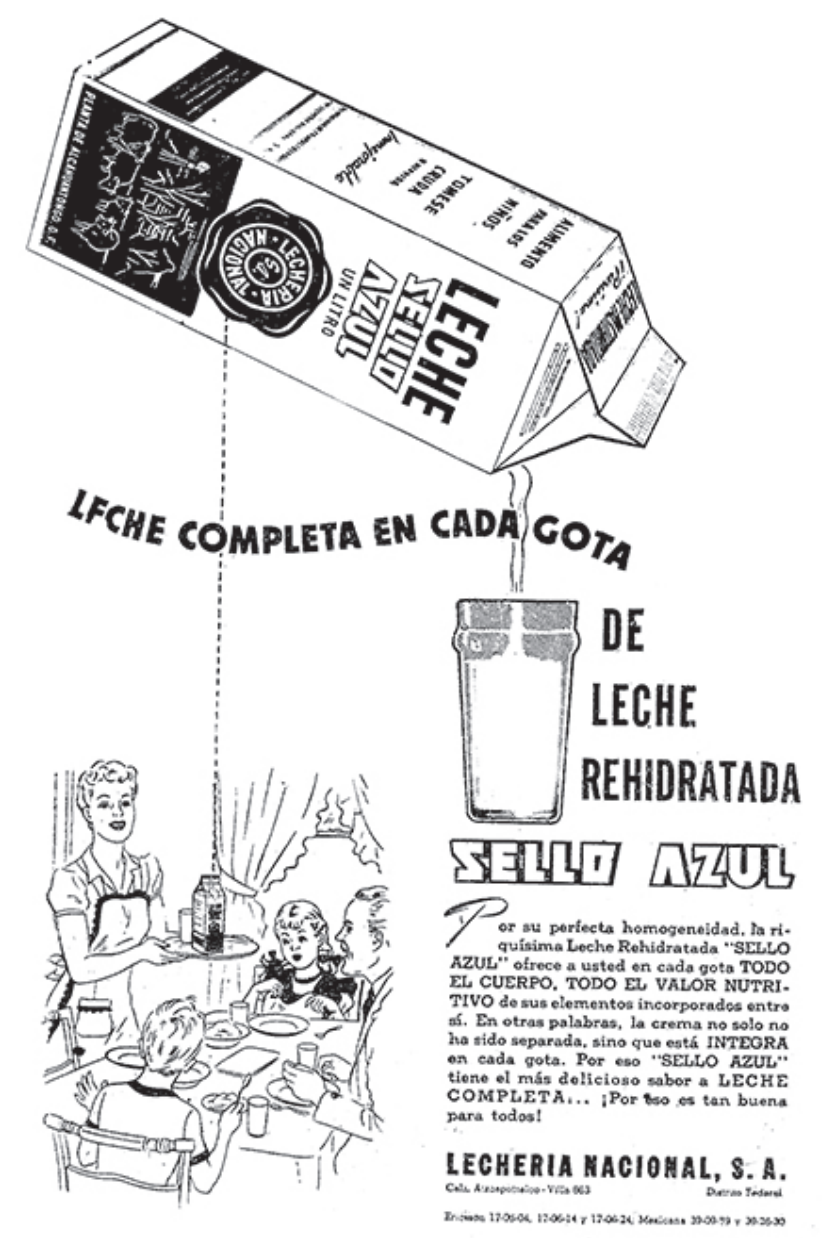

Figura 2: "Leche rehidratada Sello Azul tiene el más delicioso sabor a leche completa” (Yturbide de Limantour, 1947) 
En la imagen de la Figura 2, la madre se ve feliz y descansada a punto de servir la leche directamente del empaque ya que leche Sello Azul, por su proceso de industrialización, no requería hervirla y se podía tomar "cruda". Si bien los alimentos industrializados reducían la carga de trabajo de la mujer, no modificaban los roles de género. Por el contrario, como señala Moreno (2003), la publicidad enfatizó que la felicidad de la mujer se encontraba en la familia, el hogar y el matrimonio. La creación de Lechería Nacional también apunta a un nacionalismo económico que partía del desarrollo de la industria para incrementar el ingreso, movilidad social y prosperidad material (Moreno, 2003).

La creación de Lechería Nacional no solucionó los problemas de carestía de leche en México en parte debido a que la producción era insuficiente y la distribución limitada por falta de infraestructura como refrigeración en camiones, trenes, tiendas y centros de distribución. En 1949, una quinta parte de la leche que se vendía en la CDMX provenía de establos clandestinos que la ofertaban 15 centavos por debajo del costo de la leche en supermercados (Ochoa, 1999). Aquellos que consumían esta leche de baja calidad, y sobre todo adulterada o contaminada, eran las clases bajas quienes en consecuencia sufrían con más frecuencia de enfermedades gastrointestinales. Con el fin de mejorar la calidad de la leche que se consumía entre la clase trabajadora y reducir la venta clandestina, en 1953 se estableció otra planta rehidratadora de leche en Tlalnepantla, a las afueras de la Ciudad de México y cerca de la zona industrial donde se asentaban muchos de los campesinos que migraban a la ciudad en busca de empleo. Dicha planta dio inicio al Programa de Abasto Social de Leche con una producción de 25.000 litros al día mediante la importación de seis millones de kilos de leche semidescremada al año (del Bajío, 1990). La tendencia en la creación de plantas hidratadoras continuó en 1958 en Jiquilpan, Michoacán.

Uno de los principales centros de venta de dicha leche a precios subsidiados fue a través de las tiendas de la Compañía Exportadora e Importadora Sociedad Anónima (Ceimsa) creadas por el Estado (Azpeitia Gómez, 1994; Ochoa, 2000). Crispina y Carolina recuerdan haber comprado la leche de la Ceimsa. Si bien ellas consumieron muy poca leche durante su infancia, al ser madres y vivir en zonas más urbanas tuvieron al alcance dicho producto. Para Carolina la leche de Ceimsa no era leche de vaca, era hecha con harina de papa pues recuerda que en las ollas donde la hervía dejaba una capa que era difícil de limpiar. Aún así, consideraba que era nutritiva ya que sus hijos crecieron sanos y fuertes. A uno de sus hijos le caía mal la leche de vaca, por lo que la leche Ceimsa era la mejor opción. Crispina comenzó a comprar leche en Ceimsa en 1953, cuando tuvo su primer hijo. A veces la mezclaba con atole o café para que rindiera más, pero la leche ya era parte de la dieta de su familia. El esposo de Crispina tenía un trabajo estable como jardinero en una casa de clase alta y ella trabajaba como lavandera y costurera así que tenían un ingreso que les permitía comprar leche, algo que su mamá y sus abuelos no pudieron hacer. Ceimsa también vendía leche en polvo, por lo que en los años 1960 ella recuerda haberla comprado e hidratado en casa.

Algunas mujeres sospechaban que la leche que vendían en Ceimsa no era leche de vaca pues tenía un sabor y textura diferente. Otras encontraban la leche en polvo atractiva por su novedad. Lo que está claro es que las clases trabajadoras se acostumbraron a consumir leche reconstituida al no poder comprar leche fresca. Las historias de vida de las mujeres entrevistadas para este artículo muestran que para los años 1950 aquellas que 
no consumieron leche en su infancia ya habían aceptado la idea de la importancia de la leche, particularmente en el desarrollo infantil. Tanto Carolina como Crispina migraron a zonas urbanas de la CDMX y mejoraron su situación económica tras casarse. Para ellas, la movilidad social y física implicó un cambio en su dieta, una mejoría que se reflejó en la alimentación de sus hijos a los que trataron de dar lo que ellas no tuvieron por falta de recursos. El tomar leche se asoció con las prácticas de clase media como evidencia la publicidad de Leche Sello Azul.

El problema de la leche no se solucionó con la disponibilidad y el bajo costo. La intolerancia a la lactosa era común entre las personas de origen indígena, aunque en esta época no era identificada como tal (Lagunas Rodríguez, López Alonso, 2004). Dolores Hernández nació en 1941 en San Francisco Nuxaño, Oaxaca. Su familia no tomaba leche: “tomábamos atole de maíz, leche no. Allá no hay vacas para producir leche, en mi pueblo no hay, ni de cabra" (Hernández Santiago, 25 ago. 2005). Dolores no se definía como indígena, pero recuerda que sus padres hablaban entre ellos en una lengua indígena que ella nunca aprendió. A los 13 años migró a la CDMX y perdió contacto con la cultura de su pueblo. Dolores bebió leche por primera vez cuando trabajaba como empleada doméstica en una casa de clase media. Recuerda que no le gustaba pues le causaba diarrea, así que trataba de evitarla. Los médicos consideraban que la indigestión o diarrea causada por la leche se debía a que estaba contaminada. En el caso de los niños se recomendó el incremento paulatino de leche para que su estómago se fuera poco a poco acostumbrando a ella al ir madurando (López Fermán, 2007). La leche se mantuvo como un alimento esencial al desarrollo infantil y que debía formar parte de la dieta cotidiana del mexicano.

\section{Consideraciones finales}

El cambio en los hábitos alimenticios y el incremento del consumo de leche se vio como un elemento central para mejorar el nivel de vida de la clase obrera y campesina, ya que al ingerir más proteínas de origen animal su salud y productividad mejoraría y eventualmente el salario de los trabajadores. La leche se presentó como el alimento perfecto, esencial durante el embarazo y el desarrollo infantil. Médicos y nutriólogos reprodujeron el discurso de superioridad de la leche. Aun cuando estudios del INN mostraron que la dieta tradicional de campesinos e indígenas, basada en maíz, frijol y chile, contaba con un valor nutricional óptimo, particularmente cuando se complementaba con plantas y animales silvestres (quelites), así como insectos en algunas regiones, jamás se consideró promover dichas prácticas a nivel nacional y en muchos casos se les identificó con el atraso. Si México quería ser como los países "civilizados", debía de adoptar su dieta comenzando por el consumo de leche.

La mayoría de la leche disponible en México era reconstituida a partir de leche en polvo, evaporada o condensada importada principalmente de EEUU. La baja producción nacional, aunada a la carencia de una infraestructura y el brote de fiebre aftosa, eliminaron la posibilidad de la autosuficiencia. Mientras tanto EEUU contaba con una sobreproducción a la que debían buscarle un mercado. La FAO y la Unicef apoyaron el discurso de superioridad de la leche y equipararon la leche reconstituida con la leche fresca. La leche en polvo se 
presentó como la mejor opción particularmente para la clase trabajadora urbana. Para las mujeres que migraron del campo a la ciudad o bien que mejoraron su condición económica tras el matrimonio, el consumo de leche representó el ascenso social. La publicidad y las políticas públicas fueron dirigidas a las mujeres al considerarlas como encargadas de la alimentación familiar, lo que reprodujo percepciones de género.

El consumo de leche se vio como un de los medios para transformar a los indígenas y campesinos en mestizos. Las clases medias enfatizaron su posición al presentarse como el ejemplo a seguir ante una clase baja que debía modificar su dieta a pesar de no tener los medios económicos para lograrlo. Si bien el consumo de leche incrementó, se mantuvo lejos del ideal de un litro al día tanto por su costo como por el alto índice de intolerancia a la lactosa. Para la población de origen indígena y campesina, la leche representó metafórica y literalmente un alimento que no podían digerir. Con frecuencia se consideró que las clases bajas eran necias y obstinadas por no querer adoptar las medidas que los médicos les indicaban. Hacia los años 1980 fueron los refrescos, los alimentos procesados y altos en grasa, azúcares y carbohidratos los que vinieron a dominar la dieta de la clase trabajadora. Una modernidad que llegó con un alto precio para la salud del mexicano.

\section{NOTAS}

${ }^{1}$ En 1930 la tasa de mortalidad era de 156 niños por cada mil nacimientos (INEGI, 2001).

${ }^{2}$ La muestra se eligió a partir de amigos y conocidos por lo que en su mayoría fueron mujeres de clase media y baja. Las preguntas se centraron en las prácticas alimenticias de su infancia y las de su vida tras tener hijos. El nombre de las entrevistadas no fue modificado, con su consentimiento, esto con el fin de reconocer su agencia histórica.

${ }^{3}$ A mediados del siglo XX, clase social se definía de acuerdo al ingreso mensual de los hogares. Trescientos pesos o menos era considerado clase trabajadora, entre \$301 y \$1.500 pesos clase media baja, entre \$1.501 y $\$ 4.500$ pesos clase media y más de $\$ 4.501$ pesos clase alta. En 1963 , el $70 \%$ de la población vivía con menos de $\$ 1.500$ pesos al mes, por lo que representaba las clases bajas y medias bajas (Banco de México, 1963). En 1960, el salario mínimo en las zonas rurales era de $\$ 8,17$ al día y en las zonas urbanas de $\$ 9,41$ (Presidencia de la República, 1963).

${ }^{4}$ La leche evaporada es leche fresca a la que se le ha extraído el 60\% del agua antes de ser homogeneizada, fortificada y esterilizada. La leche condensada es una mezcla de 55-60\% de leche entera con 40-45\% de azúcar que se calienta hasta que el $60 \%$ del agua se evapora, resultando en un líquido viscoso y sumamente dulce.

${ }^{5}$ De acuerdo con dicha encuesta publicada en 1963 los poblados que tenían entre 2.501 y diez mil habitantes eran considerados urbanos en términos de población, pero rurales en relación a sus hábitos de consumo, aunque su economía no estuviera basada en la agricultura. Los pueblos con más de diez mil habitantes eran considerados urbanos (Presidencia de la República, 1963).

\section{REFERENCIAS}

AGOSTONI, Claudia. Discurso médico, cultura higiénica y la mujer en la Ciudad de México al cambio de siglo (XIX-XX). Mexican Studies/ Estudios Mexicanos, v.18, n.1, p.1-22, 2002.

AGUILAR RODRÍGUEZ, Sandra. Alimentando la nación: género y nutrición en México (19401960). Revista de Estudios Sociales, v.29, p.28-40, 2008.
AGUILAR RODRÍGUEZ, Sandra. Cooking modernity: nutrition policies, class, and gender in 1940s and 1950s Mexico City. The Americas, v.64, n.2, p.177-205, 2007.

ALVAREZ AMÉZQUITA, José et al. Historia de la salubridad y la asistencia en México. v.2. Ciudad de México: SSA, 1960. 
ARROYO, Pedro. Estudios sobre antropología y nutrición en México. In: Bertrán, Miriam; Arroyo, Pedro (org.). Antropología y nutrición. México: UAM/Nestlé, 2006. p.13-27.

AUTRET, Marcel. Informe sobre el desarrollo del programa de alimentación escolar en la Ciudad de México presentado a la Sra. de Alemán, presidenta de la Asociación ProNutrición Infantil y al Dr. Gamboa, Secretario de Salubridad y Asistencia. SubSyA, SSA, caja 29, exp.7 (Archivo Histórico de la Secretaría de Salud, Ciudad de México). 11 nov. 1951.

AZPEITIA GÓMEZ, Hugo. Compañía exportadora e importadora mexicana S.A. (1949-1958): conflicto y abasto alimentario. Ciudad de México: CIESAS, 1994.

BANCO DE MÉXICO. Encuestas sobre ingresos y gastos familiares en México. Ciudad de México: Banco de México SA-Oficina de Estudios sobre Proyecciones Agrícolas, 1963.

BARBOSA CRUZ, Mario. Distinciones y apariencias: la clase media en la Ciudad de México entre el Porfiriato y la Revolución. Oficio: Revista de Historia e Interdisciplina, v.10, p.9-23, 2020.

BASAVE MORALES, Carolina. [Entrevista.] Entrevistadora: Sandra Aguilar Rodríguez. Ciudad de México, 31 ago. 2005.

BESS, Michael K. Routes of compromise: building roads and shaping the nation in Mexico, 1917-1952. Lincoln: University of Nebraska Press, 2017.

BILTEKOFF, Charlotte. 'Strong men and women are not products of improper food': domestic science and the history of eating and identity. Journal for the Study of Food and Society, v.6, n.1, p.60-69, 2002.

BLISS, Katherine E. Compromised positions: prostitution, health and gender politics in revolutionary Mexico. Pennsylvania: Pennsylvania University Press, 2001.

BLUM, Ann S. Cleaning the revolutionary household: domestic servants and public welfare in Mexico City 1900-1935. Journal of Women's History, v.15, n.4, p.67-90, 2004.

CULLATHER, Nick. The foreign policy of the calorie. The American Historical Review, v.112, n.2, p.337-364, 2007.

DE LA PEÑA, Samuel. El grave problema de la leche. SSA, SubsyA, caja 1, exp.7 (Archivo Histórico de la Secretaría de Salud, Ciudad de México). 3 dic. 1945.

DE LA PEÑA, Samuel. Memorandum dirigido al subsecretario de la SSA emitido por el
Departamento de Producción y Provisión de leche. SSA, SubsyA, caja 1, exp.7 (Archivo Histórico de la Secretaría de Salud, Ciudad de México). 8 ene. 1945.

DE LA TORRE, José Calvo et al. Referencia especial a la nutrición popular en Las condiciones fundamentales para el progreso de la república mexicana. SSA, SubsyA, caja 17, exp.11 (Archivo Histórico de la Secretaría de Salud, Ciudad de México). abr. 1950.

DEL BAJÍO, Antonio. Crisis alimentarias y subsistencias populares en México. v.2. Ciudad de México: Leche industrializada CONASUPO SA de CV, 1990.

DGE, Dirección General de Estadística. Séptimo censo general de población. Ciudad de México: Secretaría de Economía-DGE, 1950.

DÍAZ BARRIGA, Jesús. Proyecto de programa particular relativo a la cooperación entre las Secretarías de Salubridad y Asistencia y de Educación. SSA, SubSyA, caja 17, exp.11 (Archivo Histórico de la Secretaría de Salud, Ciudad de México). 14 feb. 1950.

DÍAZ BARRIGA, Jesús. Estado de la alimentación del pueblo mexicano, sugestiones para mejorarla. SSA, SubSyA, caja 17, exp.11 (Archivo Histórico de la Secretaría de Salud, Ciudad de México). 18 nov. 1949.

DÍAZ BARRIGA, Jesús; QUINTÍN OLASCOAGA, José. Lo que come el pueblo mexicano y lo que necesita comer para estar bien nutrido: recomendaciones para orientar la producción agropecuaria de acuerdo a las necesidades nutriológicas. Salubridad y Asistencia, v.1, n.9, p.10, 1949.

DUPUIS, Melanie. Nature's perfect food: How milk became America's drink. New York: New York University Press, 2002.

EL NACIONAL, 5 ago. 1945. SSA, SubSyA, caja 3, exp.15 (Archivo Histórico de la Secretaría de Salud, Ciudad de México). 5 ago. 1945.

EL PROBLEMA de la alimentación infantil en la República Mexicana. SSA, SubSyA, caja 17, exp 11. (Archivo Histórico de la Secretaría de Salud, Ciudad de México). 1948.

ENCICLOPEDIA DEL HOGAR. Ciudad de México: Excélsior, 1946.

ESTRADA DE TREJO, Evelia. [Entrevista.] Entrevistadora: Sandra Aguilar Rodríguez. Ciudad de México, 1 sept. 2005.

EXCÉLSIOR, 12 dic. 1944. SSA, SubSyA, caja 3, exp.15 (Archivo Histórico de la Secretaría de Salud, Ciudad de México). 12 dic. 1944. 
FAO, Organización de las Naciones Unidas para la Alimentación y la Agricultura. Mejor aprovechamiento de la leche. SSA, SubSyA; caja 17, exp.11 (Archivo Histórico de la Secretaría de Salud, Ciudad de México). ago. 1949.

GUTIERREZ DEL OLMO, José Félix Alonso. De la caridad a la asistencia: un enfoque de la pobreza y la marginación en México. In: Gutiérrez del Olmo, José Félix Alonso et al. La atención materno infantil: apuntes para su historia. Ciudad de México: Secretaría de Salud, 1993. p.9-51.

HERNÁNDEZ SANTIAGO, Dolores. [Entrevista.] Entrevistadora: Sandra Aguilar Rodríguez. Ciudad de México, 25 ago. 2005.

IGUAL a la leche recién ordeñada: así será la que se traiga en polvo y se rehidrate aquí en la Capital. El Universal, 9 oct. 1945. SSA, SubSyA, caja 3, exp.15. (Archivo Histórico de la Secretaría de Salud, Ciudad de México). 9 oct. 1945.

INEGI-DGE, Instituto Nacional de Estadística, Geografía e Informática-Dirección General de Estadística. Tasas específicas de mortalidad infantil (1903-1996). Ciudad de México: INEGI-DGE, 1996.

INEGI, Instituto Nacional de Estadística, Geografía e Informática. Indicadores sociodemográficos de México (1930-2000). Ciudad de México: INEGI, 2001.

INEGI, Instituto Nacional de Estadística, Geografía e Informática. Anuarios estadísticos de las entidades federativa. Ciudad de México: INEGI, 1998.

KAPELUSZ-POPPI, Ana María. Physician activists and the development of rural health in postrevolutionary Mexico. Radical History Review, v.2001, n.80, p.35-50, 2001a.

KAPELUSZ-POPPI, Ana María. Rural health and state construction in post-revolutionary Mexico: the Nicolaita Project for Rural Medical Services. The Americas, v.58, n.2, p.261-283, 2001b.

LAGUNAS RODRÍGUEZ, Zaíd; LÓPEZ ALONSO, Sergio. Antropología física en grupos humanos de filiación Otopame. Ciencia Ergo Sum, v.11, n.1, p.47-58, 2004.

LAS CONDICIONES fundamentales para el progreso de la república mexicana. El Universal, 1950. SSA, SubSyA, caja 3, exp.15 (Archivo Histórico de la Secretaría de Salud, Ciudad de México). 1950.

LÓPEZ FERMÁN, Lilia Isabel. La leche de vaca en la dieta infantil de la Ciudad de México 1920. In: Herrera Feria, María de Lourdes (org.). Estudios sociales sobre la infancia en México. Puebla: BUAP, 2007. p.199-219.

LÓPEZ ROSADO, Diego G. Historia del abasto de productos alimenticios en la CDMX. Ciudad de México: FCE, 1988.
MARTÍNEZ BÁEZ, Manuel et al. Libro para la madre mexicana. Ciudad de México: Tipográfico La impresora, 1933

MAYORAL PARDO, Demetrio. El grave problema de la leche. SSA, SubsyA, caja 1, exp.7 (Archivo Histórico de la Secretaría de Salud, Ciudad de México). 15 abr. 1944.

MORENO, Julio. Yankee don't go home!: Mexican nationalism, American business culture, and the shaping of modern Mexico, 1920-1950. Chapel Hill: UNCP, 2003.

NESTLÉ: reflejos de setenta años en México. Ciudad de México: Gil S.A., 2001.

NIBLO, Stephen R. Mexico in the 1940s: modernity, politics, and corruption, Latin American silhouettes: studies in history and culture. Wilmington: Scholarly Resources, 1999.

OCHOA, Enrique. Feeding Mexico: the political uses of food since 1910. Wilmington: Scholarly Resources, 2000.

OCHOA, Enrique. Reappraising state intervention and social policy in Mexico: the case of milk in the Distrito Federal during the twentieth century. Mexican Studies/Estudios Mexicanos, v.15, n.1, p.73-99, 1999.

PILCHER, Jeffrey M. The sausage rebellion: public health, private enterprise, and meat in Mexico City, 1890-1917. Albuquerque: University of New Mexico Press, 2006.

PILCHER, Jeffrey M. ¡Que vivan los tamales!: food and the making of Mexican identity. Albuquerque: University of New Mexico Press, 1998.

PILCHER, Jeffrey M. Tamales or timbales: cuisine and the formation of Mexican national identity, 1821-1911. The Americas, v.53, n.2, p.193-206, 1996.

PÍO MARTÍNEZ, Juan. La ciencia de la nutrición y el control social en México en la primera mitad del siglo XX. Relaciones, v.34, n.133, p.225-255, 2013.

PRESIDENCIA DE LA REPÚBLICA. 50 años de Revolución Mexicana en cifras. Ciudad de México: Presidencia de la República-NAFINSA, 1963.

QUINTÍN OLASCOAGA, José. Datos para la historia de la nutriología en México. Salubridad y Asistencia, v.5, n.8, p.308-309, 1948.

ROSEMBLATT, Karin Alejandra. The science and politics of race in Mexico and the United States, 1910-1950. Chapel Hill: UNCP, 2018.

ROTH, Dennis. America's fascination with nutrition. Food Review, v.23, n.1, p.32-37, 2000. 
SÁNCHEZ PARRA, Cristina; MATAMOROS, María Graciela. El discreto encanto: distinción y diversión de las clases medias (México, siglos XIX y XX). Oficio: Revista de Historia e Interdisciplina, v.10, p.5-6, 2020.

SANDERS, Nichole. Gender and consumption in Porfirian Mexico: images of women in advertising 'El Imparcial,' 1897-1910. Frontiers: A Journal of Women's Studies, v.38, n.1, p.1-30, 2017.

SANDERS, Nichole. Gender and welfare in Mexico: the consolidation of a postrevolutionary state. State Park: PUP, 2011.

SAUCEDO MONTEMAYOR, Pedro. Historia de la ganadería en México. Ciudad de México: Unam, 1984.

SCHMID, Ron. The untold story of milk: green pastures, contented cows and raw dairy products. Washington: New Trends Publishing, 2003.

SE IMPORTARÁN 5 millones de latas de leche. El Nacional, 12 dic. 1944. SSA, SubSyA, caja 3, exp.15 (Archivo Histórico de la Secretaría de Salud, Ciudad de México). 12 dic. 1944.

STEPAN, Nancy Leys. The hour of eugenics: race, gender, and nation in Latin America. Ithaca: Cornell University Press, 1991.

STERN, Alexandra Minna. Eugenics in Latin America. In: Beezley, William (org.). Oxford research encyclopedia of Latin American history. Oxford: OUP, 2016.

STERN, Alexandra Minna. From mestizophilia to biotypology: racialization and science in Mexico 1920-1960 In: Appelbaum, Nancy; Macpherson, Anne; Rosemblatt, Karin (org.). Race and nation in modern Latin America. Chapell Hill: UNCP, 2003.

STERN, Alexandra Minna. Responsible mothers and normal children: eugenics, nationalism, and welfare in post-revolutionary Mexico 1920 1940. Journal of Historical Sociology, v.2, n.4, p.369-397, 1999.
SUÁREZ Y LÓPEZ GUAZO, Laura Luz. Eugenesia y racismo en México. Ciudad de México: Unam, 2005.

VARGAS DOMÍNGUEZ, Joel. El auge y declive del Instituto Nacional de Nutriología de México y su proyecto de nutrición social de 1943 a 1956. Historia Mexicana, v.69, n.2, p.511-549, 2019.

VARGAS DOMÍNGUEZ, Joel. Conexiones internacionales en fisiología, eugenesia y nutrición: las investigaciones sobre el metabolismo otomí en el México posrevolucionario. Ludus Vitalis, v.23, n.43, p.83-104, 2015.

VARGAS ROMERO, Crispina. [Entrevista.] Entrevistadora: Sandra Aguilar Rodríguez. Ciudad de México, 19 ago. 2005.

VAUGHAN, Mary Kay. Women, class, and education in Mexico, 1880-1928. Latin American Perspectives, v.4, n.1-2, p.135-152, 1977.

VAUGHAN, Mary Kay; FOWLER-SALAMINI, Heather (org.). Women of the Mexican countryside, 1850-1990: creating spaces and shaping transitions. Tucson: University of Arizona Press, 1994.

VIDAL, Faustino. Comedor familiar Num. 1. Beneficencia Pública, Establecimientos Asistenciales, Comedores Públicos, caja 6, exp.3 (Archivo Histórico de la Secretaría de Salud, Ciudad de México). 4 dic. 1941.

VIESCA TREVIÑO, Carlos. La gota de leche. De la mirada médica a la atención médicosocial en el México posrevolucionario. In: Agostoni, Claudia (ed.). Curar, sanar y educar. Enfermedad y sociedad en México, siglos XIX y $X X$. Ciudad de México: UNAM-BUAP, 2008. p.195-217.

YTURBIDE DE LIMANTOUR, Virginia. La vida social en México. Enciclopedia del Hogar. n.8. Ciudad de México: Excélsior, 1947.

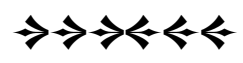

